\title{
Numeracy and Dietary Intake in Patients With Type 2 Diabetes
}

Michael E. Bowen, MD, MPH, Kerri L. Cavanaugh, MD, MHS, Kathleen Wolff, MSN, BC-ADM, BC-FNP, Dianne Davis, RD, LDN, CDE, Becky Gregory, MS, RD, LDN, CDE, and Russell L. Rothman, MD, MPP

University of Texas Southwestern Medical Center, Dallas, Texas (Dr Bowen), and the Vanderbilt University School of Medicine, Nashville, Tennessee (Dr Cavanaugh, Ms Wolff, Ms Davis, Ms Gregory, Dr Rothman)

\begin{abstract}
Purpose-The purpose of this study is to describe the association between numeracy and selfreported dietary intake in patients with type 2 diabetes.

Methods-Numeracy and dietary intake were assessed with the validated Diabetes Numeracy Test and a validated food frequency questionnaire in a cross-sectional study of 150 primary care patients enrolled in a randomized clinical trial at an academic medical center between April 2008 and October 2009. Associations between numeracy and caloric and macronutrient intakes were examined with linear regression models.
\end{abstract}

Results-Patients with lower numeracy consumed a higher percentage of calories from carbohydrates and lower percentages from protein and fat. However, no differences in energy consumption or the percentage of energy intake owing to carbohydrates, fat, or protein were observed in adjusted analyses. Patients with lower numeracy were significantly more likely to report extremely high or low energy intake inconsistent with standard dietary intake.

Conclusions-Numeracy was not associated with dietary intake in adjusted analyses. Low numeracy was associated with inaccurate dietary reporting. Providers who take dietary histories in patients with diabetes may need to consider numeracy in their assessment of dietary intake.

Diabetes self-management skills such as planning and assessing dietary intake, monitoring blood glucose, and calculating and administering medication doses are often heavily dependent on accessing, understanding, and processing complex health information. In the United States, an estimated 90 million Americans have basic or below-basic literacy skills, and 110 million have limited numeracy skills. ${ }^{1}$ Numeracy, a distinct component of health literacy, is the ability to understand and use numbers in daily life. ${ }^{2,3}$ Individuals with low health literacy may have difficulty following instructions, understanding health information, and performing self-care tasks. ${ }^{4}$ Specifically for patients with diabetes, low literacy has also been associated with worse disease knowledge and poorer clinical outcomes. ${ }^{5,6}$ In addition, lower diabetes-related numeracy has been associated with poorer diabetes self-management and worse glycemic control. ${ }^{7}$

(C) 2013 The Author(s)

Correspondence to Michael E. Bowen, MD, MPH, 5323 Harry Hines Blvd, Dallas, TX 75390-9169 (michael.bowen@utsouthwestern.edu). 
Health literacy and numeracy skills are fundamental to understanding nutrition information and can affect comprehension of nutrition labels and portion size information. ${ }^{8,9}$ Healthy eating and nutritional management are recognized by the American Diabetes Association and the American Association of Diabetes Educators as critical elements of diabetes prevention and management. ${ }^{10,11}$ Diabetes educators, registered dietitians, and other nutrition professionals play an integral role as part of the patient's diabetes care team by assessing self-care behaviors and teaching knowledge and self-management skills. ${ }^{12,13}$ Assessment of dietary intake, dietary modification, and healthy eating are critical components of both diabetes self-management education and training (DSME/T) and medical nutrition therapy (MNT). ${ }^{11,14,15}$ The American Association of Diabetes Educators recommends individualization of DSME/T and MNT on the basis of an individual's literacy and learning style. ${ }^{14,15}$ Low numeracy in particular may influence an individual's energy and macronutrient intake and pose a significant barrier to understanding and executing dietary recommendations.

A comprehensive dietary assessment is essential for the design and implementation of individualized nutrition interventions and evaluation of progress over time. ${ }^{13}$ In the research setting, the quantitative assessment of dietary intake frequently utilizes food frequency questionnaires (FFQs) because of their low cost and relative ease of administration. ${ }^{16} \mathrm{FFQs}$ ask participants to estimate portion sizes and the frequency of dietary intake of diverse food items. Concerns exist regarding respondents' accuracy in quantification of food intake on FFQs, ${ }^{16}$ and this is often reflected in reports of dietary intake that are much higher or lower than expected. Little is known about the relationship between numeracy and the accuracy of reporting on FFQ instruments.

The primary aim of this study is to describe the association among numeracy, total energy, and macronutrient intake in type 2 diabetes. A secondary aim of this study is to examine the association between numeracy and self-reports of outlying energy intake on a FFQ.

\section{Methods}

\section{Study Design}

A cross-sectional analysis was conducted on baseline data from 150 patients with type 2 diabetes who were enrolled in a randomized controlled trial between April 2008 and October 2009 that evaluated approaches to nutrition education. Patients failing to complete the FFQ $(n=6)$ were excluded. Patients were recruited from primary care clinics at the Vanderbilt University Medical Center. Eligible patients were 18 to 85 years old, English speaking, with type 2 diabetes and with most recent hemoglobin A1C greater than or equal to $7.0 \%$. Exclusion criteria included visual acuity worse than 20/50, dementia, and the use of flexible insulin dosing based on carbohydrate counting. The Vanderbilt University Institutional Review Board approved the study protocol, and all participants provided written informed consent. 


\section{Primary Predictor}

The primary exposure variable was each patient's diabetes numeracy score. Diabetes-related numeracy skills were measured with the validated 15-item Diabetes Numeracy Test (DNT) at the time of enrollment. ${ }^{17}$ The DNT assesses an individual's ability to apply basic math skills to solve nutrition and diabetes self-care calculations, including interpretation of nutrition labels and calculation of insulin doses. The DNT was administered by a trained research assistant. Patients were allowed to use calculators as needed, and no time limit for completion of the test was imposed. The DNT was scored as the percentage of items answered correctly (range, $0 \%-100 \%$ ) with missing items considered incorrect responses.

\section{Primary Outcome}

The primary outcome was the percentage of daily energy intake from carbohydrates. Carbohydrate intake was chosen as the primary outcome because of the frequent focus on carbohydrate intake in DSME/T and MNT education about healthy eating. Secondary outcomes included total energy intake in kilocalories per day $(\mathrm{kcal} / \mathrm{d})$ and the percentage of daily energy intake from protein and fat. Dietary intake was measured by the Block Brief 2000 Food Frequency Questionnaire (Block FFQ) ${ }^{18}$ which was modified to assess dietary intake in the past 30 days. The Block FFQ assesses dietary intake of 60 food items, requires approximately 17 minutes to administer in an interview setting, and has been validated. ${ }^{19}$ The Block FFQ was administered in paper format by a trained research assistant. Block FFQ forms were subsequently analyzed and converted to estimated average daily macronutrient intakes by Nutritionquest (Berkeley, California). ${ }^{18}$ As in previous studies using FFQ data, men and women reporting implausible total energy intake on the Block FFQ were excluded from this analysis $(<800$ or $>4200 \mathrm{kcal} / \mathrm{d}$ for men, $\mathrm{n}=7 ;<500$ or $>3500 \mathrm{kcal} / \mathrm{d}$ for women, $\mathrm{n}=8) .^{20,21}$

Other variables, including patient characteristics and hemoglobin A1C, were collected by patient self-report and medical record review by a trained research assistant. Health literacy was evaluated with the Rapid Estimate of Adult Learning in Medicine. ${ }^{22}$

\section{Statistical Analysis}

Given the small sample size and nonnormal distribution of data, nonparametric analyses were utilized to calculate descriptive and bivariate statistics, including median and interquartile range (IQR). Rank-sum, Pearson $\chi^{2}$, or Fisher exact tests were used as appropriate. For bivariate analyses, as in previous analyses, patients were dichotomized by DNT score comparing the lowest quartile of numeracy score with the remaining quartiles. ${ }^{7}$

The association between continuous DNT score and percentage daily carbohydrate intake was assessed with multivariate linear regression models adjusting a priori for age (continuous), sex, race (white vs nonwhite), and annual income ( $<20000$ vs $\geq \$ 20000$ ). Education and literacy were excluded from models owing to a high degree of collinearity and the potential for overadjustment. ${ }^{23}$ These models were also applied for secondary outcomes of total energy intake and percentage daily energy intake due to protein and fat. A $\log$ transformation of total daily caloric intake was performed for regression analysis because of its nonnormal distribution; however, results were back-transformed into 
kilocalories per day for reporting. Results of multivariate linear regression models were reported as a change in intake associated with an absolute $25 \%$ decrease in DNT score. Statistical significance was defined as $P<.05$. Statistical analyses were conducted with Stata 10.0 (StataCorp, College Station, Texas).

\section{Analysis of Numeracy and the Accuracy of FFQ Reporting}

Given that low numeracy has been associated with inaccurate portion size estimation, ${ }^{8}$ the relationship between numeracy and the report of extreme caloric intake ${ }^{20,21}$ was also examined to investigate possible reporting inaccuracies on the FFQ. The characteristics of patients excluded from the primary analysis $(n=15)$, by reporting extreme energy intake on the Block FFQ, were assessed with descriptive statistics. In a logistic regression model involving all 144 participants, the relationship between DNT score and the odds of reporting extreme caloric intake was examined and adjusted for the same covariates noted above.

\section{Results}

Of 150 patients participating in the study, 144 (96\%) completed a baseline Block FFQ and were included in this analysis. Patients failing to complete Block FFQs were younger (median age, 44; IQR, 34-46) than those who did complete it (median, 56; IQR, 48-62; $P$ $=.03]$ and more likely to be of nonwhite race $(83 \%$ vs $33 \%, P=.02)$. No difference in median DNT score was observed between patients completing and not completing the Block FFQ $(77 \%$ vs $67 \%, P=.53)$.

Patient characteristics by numeracy status and caloric intake are shown in Table 1 . The median age of the study sample was 56 years (IQR, 48-62). Two-thirds of study participants were white and just over half were women. Over $75 \%$ of study participants were high school graduates, and over $90 \%$ had a health literacy level at the ninth-grade level or higher. The median score on the DNT was 67\% (IQR, 43\%-87\%). Study participants had a median A1C of $8.3 \%$ (IQR, 7.5\%-9.9\%) at baseline. The median duration of diabetes was 8 years (IQR, 3-12), and one-third of patients used insulin. Patients reported a median caloric intake of $1582 \mathrm{kcal} /$ day (IQR, 1131-2160) with a median of 42\% of daily energy intake from carbohydrates (IQR, 37\%-50\%).

Among those not reporting extreme caloric intake $(n=129)$, patients with lower numeracy were significantly more likely to be older, nonwhite, and women, with less than a high school education, with an annual income less than $\$ 20000$, and with less than a ninth-grade health literacy level (Table 1). Patients with lower numeracy reported a lower daily energy intake than did patients with higher numeracy (1428 vs $1703 \mathrm{kcal} / \mathrm{day}, P=.20)$; however, this difference was not statistically significant. Patients with lower numeracy consumed higher percentages of daily caloric intake from carbohydrates $(47 \%$ vs $42 \%, P=.008)$ and lower percentages of dietary intake from protein $(16 \%$ vs $17 \%, P=.015)$ and fat $(40 \%$ vs $42 \%, P=.048)$ compared with individuals with higher numeracy in unadjusted analyses (Table 1). After adjustment for age, sex, race, and income, diabetes numeracy score was not associated with a difference in daily caloric intake or the percentage of daily energy intake from carbohydrates, fat, or protein (Table 2). 
Given the uncertainty about the accuracy of dietary intake reporting in patients with lower numeracy, patients who were excluded from analysis according to the a priori definition of extreme energy intake ${ }^{20,21}$ were examined separately (Table 1). Patients reporting extreme caloric intake ( $\mathrm{n}=15)$ were more likely to be nonwhite $(67 \%$ vs $29 \%, P=.01)$ and have lower median DNT scores: 40\% (IQR, 20\%-67\%) versus 73\% (47\%-87\%; $P=.02)$. Equal numbers of patients reported extremely low intake ( $\mathrm{n}=7$; median, $659 \mathrm{kcal} / \mathrm{day}$; IQR, 483721 ) and extremely high intake ( $\mathrm{n}=8$; median, $4483 \mathrm{kcal} / \mathrm{d}$; IQR, 3915-5553). Patients with lower numeracy were more likely to report extreme energy intake (odds ratio $=27 ; 95 \%$ confidence interval: 3,$210 ; P=.002$ ). This relationship persisted after adjustment for age, sex, race, and income (odds ratio $=17 ; 95 \%$ confidence interval: $1.6,185 ; P=.02$ ).

\section{Discussion}

In this study, individuals with lower numeracy consumed a greater proportion of their daily caloric intake from carbohydrates than individuals with higher numeracy; however, after adjustment for potential confounders, no association between numeracy and total energy or macronutrient intake was observed. Individuals with lower numeracy were more likely to report extreme caloric intake on the FFQ, raising concerns about the accuracy of dietary intake reports in diabetes patients with lower quantitative skills.

Although dietary management of diabetes is numeracy intensive and individuals with low numeracy struggle to accurately interpret food labels and estimate portion sizes, ${ }^{8,9}$ the anticipated association between low numeracy and reporting increased caloric and carbohydrate intake was not observed. This may be due to the small sample size, with lack of sufficient power, or in part related to residual confounding by complex psychological and societal influences on eating behaviors not accounted for. Additionally, high or low dietary intakes may be independent of numeracy status, especially when individuals are not actively engaged in dietary monitoring and self-management. Study participants also may have altered their dietary reporting on the basis of the perceived social desirability of their responses ${ }^{24}$ and knowledge of participating in a diabetes education study.

A healthy diet focused on appropriate caloric intake is an important element of diabetes prevention and treatment. MNT emphasizes the importance of energy balance as a critical determinant of weight loss, weight maintenance, and diabetes management. ${ }^{10}$ In this study, energy intake did not differ by numeracy status. Participants reported a median caloric intake of $1575 \mathrm{kcal}$ per day, which is similar to the energy intake reported in other diabetes nutrition studies ${ }^{25,26}$ and studies suggesting that FFQs may underestimate energy intake. ${ }^{16,27}$ Although energy intake is not typically quantified in MNT or DMSE/T, energy and macronutrient intake reported on interviewer-administered dietary histories are comparable with reports on FFQs and 7-day dietary recalls. ${ }^{28}$ Given the high frequency of dietary underreporting on FFQs, underestimation and underreporting of dietary intake during routine DMSE/T and MNT nutrition assessments may be common.

While the optimal distribution of macronutrient intake in the diet of patients with diabetes is unknown, participants in this study reported dietary intake of carbohydrates, protein, and fat similar to those recommended by the dietary reference intakes. ${ }^{29}$ Individuals with lower 
numeracy reported significantly higher energy intake from carbohydrates in unadjusted analyses when compared with those with higher numeracy. Although this difference did not persist after adjustment for confounders, the association between low numeracy and basic nutrition skills ${ }^{8,9}$ may limit successful implementation of nutrition recommendations in individuals with low numeracy. Given that carbohydrate monitoring is critical to the nutritional management of diabetes, ${ }^{10}$ these patients may benefit from simplified methods of carbohydrate monitoring, such as the plate method. ${ }^{30}$

Dietary reporting requires numeracy skills to assess, interpret, and report portion size and frequency estimates of food consumption. The ability of patients with low numeracy to accurately report dietary intake, both in dietary interviews and on quantitative assessments such as the FFQ, is instrumental to DSME/T and MNT. ${ }^{13}$ Although dietary history and FFQs are often conducted orally in an attempt to circumvent limited print literacy skills among respondents, this approach is unlikely to address low numeracy skills. Individuals with low socioeconomic status, ${ }^{31}$ less than a high school education,,${ }^{32,33}$ obese individuals, ${ }^{27}$ and female respondents ${ }^{34}$ tend to have greater inaccuracies in dietary reporting on FFQs. Portion size has been associated with sex-related inaccuracies in dietary reporting. Although the Block FFQ accounts for portion size, its abbreviated food item list may underestimate sex differences in energy intake. ${ }^{35}$ In this study, individuals with low numeracy had characteristics similar to those of individuals with low numeracy in other studies 7,36 and were significantly more likely to report extreme energy intake, suggesting inaccurate reporting of dietary intake. ${ }^{37}$ However, these results should be interpreted with caution given the secondary analysis, small sample size, and lack of a 24-hour dietary recall as a gold standard for comparison.

While alternate approaches to dietary assessment in the elderly and low income individuals have been examined, ${ }^{34,38}$ few FFQs have been validated in participants with lower literacy or numeracy skills. Validation studies of most FFQs have been conducted primarily with well-educated white men and women, and the validity of these instruments in socially and educationally disadvantaged populations is unknown. ${ }^{34}$ Analytic approaches that exclude individuals reporting extreme caloric intake from analysis may systematically exclude individuals with lower numeracy from nutritional analyses and introduce bias. Further study of health literacy and numeracy in dietary assessment may help explain variation in dietary reporting.

\section{Implications for Practice}

Multiple aspects of MNT and DSME/T—including dietary assessment, carbohydrate and calorie counting, and execution of dietary recommendations-are heavily dependent on numeracy skills. Although low numeracy is associated with inaccurate portion size estimation, misinterpretation of food labels, and worse diabetes self-care, an association between numeracy and total energy intake or carbohydrate intake was not observed in this study. However, given the common use of numbers in DSME/T and MNT, additional research is needed in larger patient populations to determine the role of numeracy in DSME/T and MNT. 
The American Association of Diabetes Educators recommends individualization of DSME/T and MNT based on an individual's health literacy, learning style, and self-care skills; however, successful individualization of nutrition education depends on an accurate assessment of dietary intake. This study suggests that individuals with low numeracy are more likely to report energy intakes outside the expected range. This may reflect numeracyrelated challenges in reporting dietary intake. Although numeracy is not routinely measured in clinical practice, education level, print literacy, and verbal literacy skills are often used as proxies for numeracy. However, such approaches may be misleading when one attempts to assess dietary intake in DSME/T and MNT, as patients may have adequate verbal literacy skills but limited numeracy skills and difficulty performing basic math calculations. ${ }^{2,7}$ Individuals reporting low caloric intake may still struggle to lose weight as a result of underestimation of dietary intake and overestimation of expenditure. ${ }^{39}$ This observation may be mediated by low numeracy and consideration of numeracy in the assessment of dietary history and quantification of dietary intake may be important.

Additionally, numeracy may influence the ability of individual patients to understand and successfully implement DMSE/T and MNT recommendations. Literacy- and numeracysensitive approaches to DMSE/T and MNT have been developed and shown to improve glycemic control. ${ }^{30,36}$ The development of numeracy-sensitive approaches to dietary assessment may further improve not only the measurement of dietary intake but also the effectiveness of DMSE/T and MNT. Providers who assess dietary intake in patients with diabetes may need to consider numeracy when taking dietary histories, measuring dietary intake, and providing dietary recommendations.

\section{Acknowledgments}

This study was funded by the American Association of Diabetes Educators and Research Foundation (RFA-0100-06) and the Vanderbilt Institute for Clinical and Translational Research (NIH UL1 RR024975-01). Dr Bowen is supported by the VA National Quality Scholars Fellowship. Dr Cavanaugh is supported by NIH/NIDDK K23 DK080952.

\section{References}

1. Kutner, MGE.; Ying, J.; Boyle, B.; Hsu, Y.; Dunleavy, E.; White, S. Literacy in Everyday Life: Results From the 2003 National Assessment of Adult Literacy. Washington, DC: National Center for Education Statistics; 2007.

2. Rothman RL, Montori VM, Cherrington A, Pignone MP. Perspective: the role of numeracy in health care. J Health Commun. 2008; 13(6):583-595. [PubMed: 18726814]

3. Nielsen-Bohlman, LPA.; Kindig, DA., editors. Health Literacy: A Prescription to End Confusion. Washington, DC: National Academies Press; 2004.

4. Schillinger D, Grumbach K, Piette J, et al. Association of health literacy with diabetes outcomes. JAMA. 2002; 288(4):475-482. [PubMed: 12132978]

5. Rothman R, Malone R, Bryant B, Horlen C, DeWalt D, Pignone M. The relationship between literacy and glycemic control in a diabetes disease-management program. Diabetes Educ. 2004; 30(2):263-273. [PubMed: 15095516]

6. Rothman RL, Malone R, Bryant B, et al. The Spoken Knowledge in Low Literacy in Diabetes Scale: a diabetes knowledge scale for vulnerable patients. Diabetes Educ. 2005; 31(2):215-224. [PubMed: 15797850]

7. Cavanaugh K, Huizinga MM, Wallston KA, et al. Association of numeracy and diabetes control. Ann Intern Med. 2008; 148(10):737-746. [PubMed: 18490687] 
8. Huizinga MM, Carlisle AJ, Cavanaugh KL, et al. Literacy, numeracy, and portion-size estimation skills. Am J Prev Med. 2009; 36(4):324-328. [PubMed: 19285197]

9. Rothman RL, Housam R, Weiss H, et al. Patient understanding of food labels: the role of literacy and numeracy. Am J Prev Med. 2006; 31(5):391-398. [PubMed: 17046410]

10. Bantle JP, Wylie-Rosett J, Albright AL, et al. Nutrition recommendations and interventions for diabetes: a position statement of the American Diabetes Association. Diabetes Care. 2008; 31(suppl 1):S61-S78. [PubMed: 18165339]

11. American Association of Diabetes Educators. Healthy eating: incorporating nutritional management into lifestyle. Diabetes Educ. 2012; 38(1):124-128. [PubMed: 22328038]

12. Martin, C.; Daly, A.; McWhorter, LS.; Shwide-Slavin, C.; Kushion, W.; American Association of Diabetes Educators. The scope of practice, standards of practice, and standards of professional performance for diabetes educators. http://www.diabeteseducator.org/export/sites/aade/ _resources/pdf/research/Diabetes_Educator_ScopeStandards_Final2-1-11.pdf. Accessed Febuary 15,2012

13. Morris S, Wylie-Rosett J. Medical nutrition therapy: a key to diabetes management and prevention. Clin Diabetes. 2010; 28:12-18.

14. Funnell MM, Brown TL, Childs BP, et al. National standards for diabetes self-management education. Diabetes Care. 2010; 33(suppl 1):S89-S96. [PubMed: 20042780]

15. American Association of Diabetes Educators. AADE position statement: individualization of diabetes self-management education. Diabetes Educ. 2007; (33):45-49. [PubMed: 17272792]

16. Thompson, F.; Subar, A. Nutrition in the Prevention and Treatment of Disease. Bethesda, MD: National Cancer Institute; 2012. Assessment methods for research and practice.

17. Huizinga MM, Elasy TA, Wallston KA, et al. Development and validation of the Diabetes Numeracy Test (DNT). BMC Health Serv Res. 2008; 8:96. [PubMed: 18452617]

18. Block Brief 2000 Food Frequency Questionnaire. http://www.nutritionquest.com. Accessed August 10,2011

19. Block G, Hartman AM, Naughton D. A reduced dietary questionnaire: development and validation. Epidemiology. 1990; 1(1):58-64. [PubMed: 2081241]

20. Willett, W. Nutritional Epidemiology. 2. New York, NY: Oxford University Press; 1998.

21. Joshipura KJ, Hu FB, Manson JE, et al. The effect of fruit and vegetable intake on risk for coronary heart disease. Ann Intern Med. 2001; 134(12):1106-1114. [PubMed: 11412050]

22. Davis TC, Long SW, Jackson RH, et al. Rapid estimate of adult literacy in medicine: a shortened screening instrument. Fam Med. 1993; 25(6):391-395. [PubMed: 8349060]

23. DeWalt DA, Pignone MP. Reading is fundamental: the relationship between literacy and health. Arch Intern Med. 2005; 165(17):1943-1944. [PubMed: 16186462]

24. Horner NK, Patterson RE, Neuhouser ML, Lampe JW, Beresford SA, Prentice RL. Participant characteristics associated with errors in self-reported energy intake from the Women's Health Initiative Food-Frequency Questionnaire. Am J Clin Nutr. 2002; 76(4):766-773. [PubMed: 12324289]

25. Vitolins MZ, Anderson AM, Delahanty L, et al. Action for Health in Diabetes (Look AHEAD) trial: baseline evaluation of selected nutrients and food group intake. J Am Diet Assoc. 2009; 109(8):1367-1375. [PubMed: 19631042]

26. Jarvandi S, Gougeon R, Bader A, Dasgupta K. Differences in food intake among obese and nonobese women and men with type 2 diabetes. J Am Coll Nutr. 2011; 30(4):225-232. [PubMed: 21917702]

27. Livingstone MB, Black AE. Markers of the validity of reported energy intake. J Nutr. 2003; 133(suppl 3):895S-920S. [PubMed: 12612176]

28. Jain M, Howe GR, Rohan T. Dietary assessment in epidemiology: comparison on food frequency and a diet history questionnaire with a 7-day food record. Am J Epidemiol. 1996; 143(9):953-960. [PubMed: 8610709]

29. Otten, JJ.; Hellwig, JP.; Meyers, LD., editors. Dietary Reference Intakes: The Essential Guide to Nutrient Requirements. Washington, DC: National Academies Press; 2006. 
30. Wolff K, Cavanaugh K, Malone R, et al. The Diabetes Literacy and Numeracy Education Toolkit (DLNET): materials to facilitate diabetes education and management in patients with low literacy and numeracy skills. Diabetes Educ. 2009; 35(2):233-236. 238-241, 244-235. [PubMed: 19240246]

31. Stallone DD, Brunner EJ, Bingham SA, Marmot MG. Dietary assessment in Whitehall II: the influence of reporting bias on apparent socioeconomic variation in nutrient intakes. Eur J Clin Nutr. 1997; 51(12):815-825. [PubMed: 9426356]

32. Kristal AR, Feng Z, Coates RJ, Oberman A, George V. Associations of race/ethnicity, education, and dietary intervention with the validity and reliability of a Food Frequency Questionnaire: the Women's Health Trial Feasibility Study in Minority Populations. Am J Epidemiol. 1997; 146(10): 856-869. [PubMed: 9384206]

33. Mayer-Davis EJ, Vitolins MZ, Carmichael SL, et al. Validity and reproducibility of a food frequency interview in a Multi-Cultural Epidemiology Study. Ann Epidemiol. 1999; 9(5):314324. [PubMed: 10976858]

34. Quandt SA, Vitolins MZ, Smith SL, et al. Comparative validation of standard, picture-sort and meal-based food-frequency questionnaires adapted for an elderly population of low socioeconomic status. Public Health Nutr. 2007; 10(5):524-532. [PubMed: 17411474]

35. Molag ML, de Vries JH, Ocke MC, et al. Design characteristics of food frequency questionnaires in relation to their validity. Am J Epidemiol. 2007; 166(12):1468-1478. [PubMed: 17881382]

36. Cavanaugh K, Wallston KA, Gebretsadik T, et al. Addressing literacy and numeracy to improve diabetes care: two randomized controlled trials. Diabetes Care. 2009; 32(12):2149-2155. [PubMed: 19741187]

37. Poslusna K, Ruprich J, de Vries JH, Jakubikova M, van't Veer P. Misreporting of energy and micronutrient intake estimated by food records and 24 hour recalls, control and adjustment methods in practice. Br J Nutr. 2009; 101(suppl 2):S73-S85. [PubMed: 19594967]

38. Fornes NS, Stringhini ML, Elias BM. Reproducibility and validity of a Food-Frequency Questionnaire for use among low-income Brazilian workers. Public Health Nutr. 2003; 6(8):821827. [PubMed: 14641954]

39. Lichtman SW, Pisarska K, Berman ER, et al. Discrepancy between self-reported and actual caloric intake and exercise in obese subjects. N Engl J Med. 1992; 327(27):1893-1898. [PubMed: 1454084] 


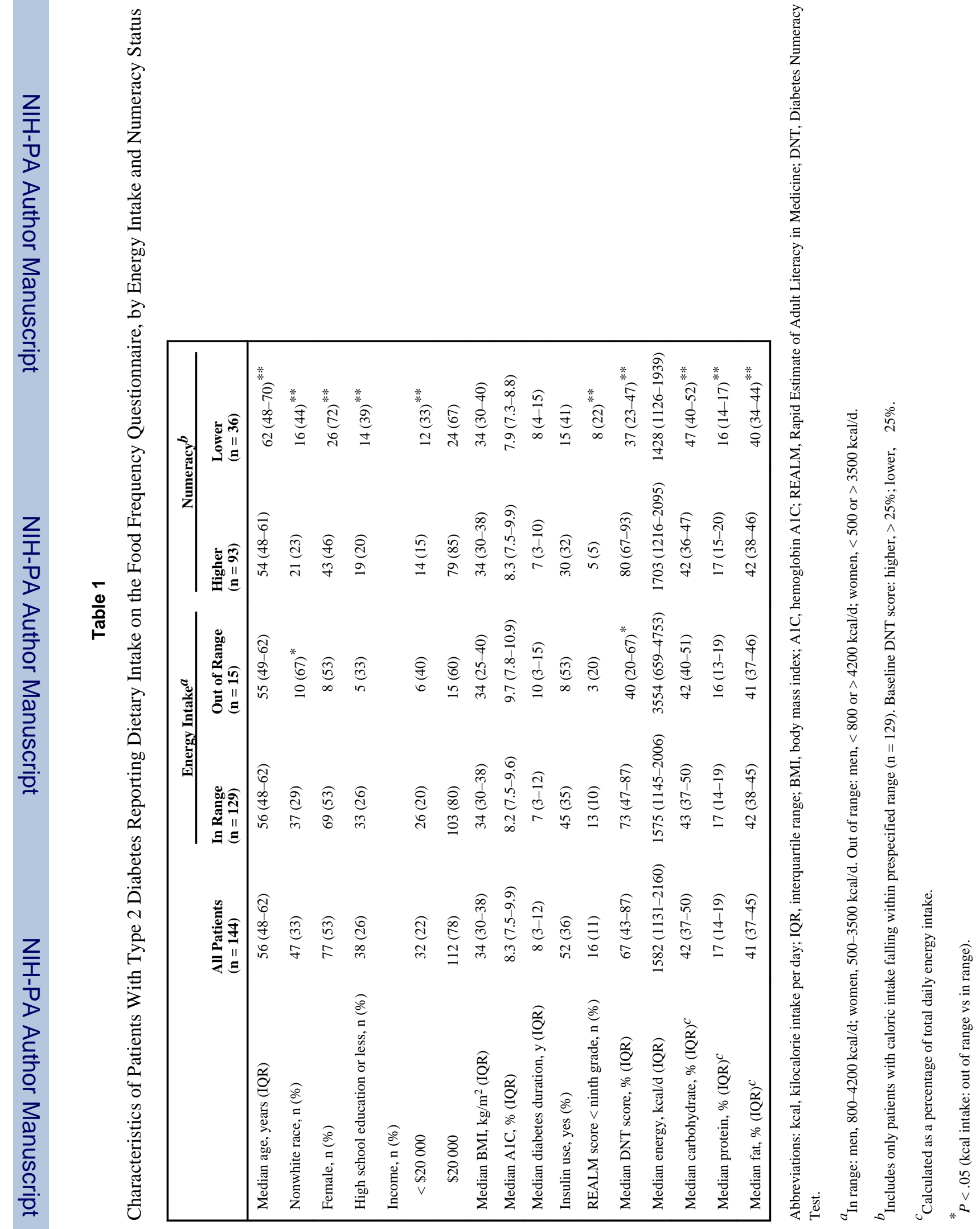




\section{Table 2}

Change in Nutritional Intake Measured on the Block-Brief Food Frequency Questionnaire Associated With a 25\% Decrease in Diabetes Numeracy Test Score

\begin{tabular}{|lrcl|}
\hline Daily Nutrient Intake & \multicolumn{1}{l}{$\boldsymbol{\beta}$} & Change $\left(\mathbf{9 5 \%}\right.$ CI) $^{\boldsymbol{a}}$ & $\boldsymbol{P}$ \\
\hline Total kcal/day & 0.97 & $24(-19,32)$ & .82 \\
Carbohydrate, $\% b$ & 5.3 & $1.3(-0.3,3.0)$ & .12 \\
Protein, $\%^{b}$ & -2.8 & $-0.7(-1.4,0.02)$ & .06 \\
Fat, $\% b$ & -2.2 & $-0.6(-1.9,0.74)$ & .40 \\
\hline
\end{tabular}

${ }^{a}$ Linear regression model including patients with daily energy intake in range $(\mathrm{n}=129)$; models adjusted for age, sex, race, and income.

${ }^{b}$ Calculated as a percentage of total daily energy intake. CI, confidence interval. 